Check for updates

Cite this: RSC Adv., 2017, 7, 49655

Received 28th September 2017 Accepted 19th October 2017

DOI: 10.1039/c7ra10759e

rsc.li/rsc-advances

\section{Characterization of the adsorption dynamics of trisodium citrate on gold in water solution $\uparrow$}

\author{
Susanna Monti, (D) *a Giovanni Barcaro, ${ }^{\text {b }}$ Luca Sementa, ${ }^{\text {b }}$ Vincenzo Carravetta (D) b \\ and Hans Ågren (D) cd
}

Molecular dynamics simulations (MDs) based on a reactive force field (ReaxFF) are carried out to investigate typical adsorption modes of trisodium citrate (CIT) on gold nanoparticles (AuNPs). All possible configurations of CIT on a model AuNP in solution are identified and the stabilizing role played by the adsorbate on the AuNP structure is disclosed by analyzing protonation state, dynamics and interactions of the molecules with the surrounding environment. A realistic scenario is created by sampling extensively the conformational space of citrate and by validating the simulation results against data extracted from the literature. Carboxyl moieties are found in contact with the gold substrate through one or both oxygens and $\mathrm{Na}^{+}$ions are present in the adsorbed citrate layer. Citrate binding is relatively strong and its action as a chelator stabilizes surface reconstructions. The molecules have the tendency to self-assemble and form a stable cover, which is reinforced by adatoms. All the results are in perfect agreement with experimental observations and theoretical data.

\section{Introduction}

Over the last few years a great variety of experimental and theoretical investigations have focused on gold-based nanomaterials for developing effectively tuned cancer therapies, local drug delivering agents, efficient detectors and specific biosensors. ${ }^{\mathbf{1}-4}$ All these studies tried to disclose, at the nanoscale level, structure, dynamics and physicochemical properties of different types of hybrid systems in order to suggest modifications and new design strategies to improve their performance. Gold nanoparticles (AuNPs) are very promising vehicles, efficient radio-sensitisers and can be used as photothermal and contrast agents. ${ }^{5,6}$

The selection of specific AuNPs morphologies is fundamental for the target application and it is often made on the basis of the nanoparticle dynamics and response. For example, due to the enhanced signals, spiky structures and irregular shapes are preferred to spherical conformations for in vivo experiments, whereas spherical forms are the ideal choice for

${ }^{a}$ CNR-ICCOM, Institute of Chemistry of Organometallic Compounds, via G. Moruzzi 1, I-56124 Pisa, Italy.E-mail: sapeptides@gmail.com

${ }^{b}$ CNR-IPCF, Institute of Chemical and Physical Processes, via G. Moruzzi 1, I-56124 Pisa, Italy

'KTH Royal Institute of Technology, School of Biotechnology, Division of Theoretical Chemistry and Biology, S-106 91 Stockholm, Sweden

${ }^{d}$ Institute of Nanotechnology, Spectroscopy and Quantum Chemistry, Siberian Federal University, Svobodny pr. 79, 660041 Krasnoyarsk, Russia

$\dagger$ Electronic supplementary information (ESI) available: Citrate speciation plot, potential energy trend during equilibration/production, $\mathrm{Au}-\mathrm{O}$ minimum distance distribution. See DOI: 10.1039/c7ra10759e immune-gold labeling and lateral flow tests. ${ }^{7-10}$ As a matter of fact, specific AuNP structures can be obtained by biasing the first steps of their preparation with suitable substances and by modulating the environmental conditions. This has been reported in a large number of books, reviews and articles, where production, properties and applications are exhaustively described (see ref. 11-19 and references therein).

All of these papers agree in defining the seed-mediated growth method as one of the most widespread techniques for synthesizing the nanoparticles, with a thorough control of their shape and size, and citrate as one of the leading compounds (citric acid reduction reactions) and stabilizing agent. The role played by citrate is recognized and attributed to its electrostatic attributes. ${ }^{20}$ Indeed, the stabilization is essentially due to the mutual repulsion between neighboring AuNPs that is caused by the negative surface charge of the citrate layer. According to earlier publications, the layer thickness is around $0.4-0.7 \mathrm{~nm}$ (ref. 21) and the citrate-to-gold ratio ${ }^{22}$ can be changed to adjust the size of the final carriers.

Disclosing the intermolecular interactions (gold-adsorbate and adsorbate-adsorbate interactions) together with the adsorbates structure and dynamics at the interface is very important for correlating these features with the changes in the physicochemical properties of the nanoparticle materials and their stability in solution. ${ }^{23,24}$

By means of classical molecular dynamics based on ReaxFF $^{25-29}$ we depict comprehensively citrate adsorption on gold by including explicit solvent molecules (water and sodium counterions), a realistic representation of the metal support (a $3 \mathrm{~nm}$ AuNP), a coating multilayer, reactivity and dynamics of 
all the species. The behavior in solution of the model AuNP coated with at least a monolayer of citrate molecules is disclosed considering both acidic and basic conditions. No restraints are introduced and all species can interact with each other and modify their conformation in response to the environment. This is an application of the methodology we have developed and used successfully in previous studies. ${ }^{30-33}$

\section{Computational details}

\section{Model building and system setup}

The coordinates of the metal support were extracted from an earlier study ${ }^{32}$ where the adsorption mechanism of cysteine onto an AuNP in solution was investigated exhaustively. The chosen model was an equilibrated structure composed of 887 $\mathrm{Au}$ atoms with a truncated octahedron shape. Its maximum diameter was around $3 \mathrm{~nm}$ and the low index (111), (100) and (110) facets were all available for binding. As already observed, ${ }^{32}$ due to the size of the system, the extension of these regions was limited and thus each of them could host three or four molecules at most. In order to decorate the surface randomly, the AuNP was surrounded by a shell of sparse citrate units (90 in all), which were sufficient to cover the whole interface. These molecules were placed relatively far from the AuNP (with minimum distance around $6 \AA$ ) so that the added water could penetrate the enveloping citrate layer and reach the surface. Then, the complex was solvated by water molecules (around 14000 ) and $\mathrm{Na}^{+}$counterions (total number of atoms in the system: 43 551). Acidic and basic $\mathrm{pH}$ conditions were simulated considering two different ideal starting configurations: one where all the citrates were $(-3)$ anions and the other one where all the molecules were doubly deprotonated $(-2)$. In both cases they were neutralized by the required number of $\mathrm{Na}^{+}$counterions (270 or 180). According to the table reported in ref. 34 at a $\mathrm{pH}=7$ citric acid is nearly completely dissociated ( -3 anions), whereas at acidic $\mathrm{pH}$ all the other species prevail. ${ }^{34}$ The final simulation box after equilibration was about $8 \times 8 \times 8 \mathrm{~nm}^{3}$.

The starting conformation of citric acid was obtained from a DFT/B3LYP/6-311++G(d,p) level optimization in the gas phase. Minimum energy structures and torsional energy barriers necessary to describe correctly this type of molecule were included in the original parametrization of the protein force field and tested for several different structures. ${ }^{29}$

Reactive molecular dynamics simulations based on the force field developed in ref. 31 were performed, first, in the NVT ensemble at $T=300 \mathrm{~K}$ for about $50 \mathrm{ps}$ and then in the NPT ensemble for about 50 ps to get the correct density. The whole time period (around $100 \mathrm{ps}$ ) was sufficient to equilibrate the system at the selected temperature. In this phase, almost all the molecules (around 40\%) that were in a favorable orientation settled on the surface, whereas the other $60 \%$, more solvated and interconnected by hydrogen bonds, remained in the outer regions. Their motion toward the support and conformational rearrangement was slower but was completed in the subsequent $100 \mathrm{ps}$ of the production stage, which was carried out in the NVT ensemble. These initial steps were not included in the final analysis. The sampling phase started afterwards and was extended for 1000 more picoseconds. System snapshots were saved every $0.025 \mathrm{ps}$ and a total of 3600000 configurations of the citric acid molecule were collected and analyzed. Temperature was controlled through the Berendsen's thermostat ${ }^{35}$ with a relaxation constant of $0.1 \mathrm{ps}$ and the time step was set to 0.25 fs. The trajectories were examined focusing on species, adsorption modes, molecular conformations, coordination numbers, atom-atom radial distribution functions (RDFs) and various types of distances. In order to analyze the protonation states of the citrate molecules an O-H cutoff distance of $1.15 \AA$ was considered. The Amsterdam Density Functional (ADF) ${ }^{36}$ and LAMMPs $\operatorname{codes}^{37}$ were employed for all the simulations.

\section{Results and discussion}

\section{Molecular species}

In the final configuration extracted from the MD trajectories, the AuNP surface is partially covered (60\% at most) with adsorbed citrate molecules that adopt a great variety of orientations. These were classified according to the connections of the carboxyl oxygens to the interface, namely monodentate and bidentate coordinations or a combination of the two (Fig. 1 and 2 ). The binding modes were directly correlated not only to the final species existent in the system that evolved just because of the chosen general conditions $(\mathrm{pH})$, but also to local reorganizations due to surface reconstructions, citrate self-interactions, sodium ions relocations and perturbations induced by water. Before discussing the type of adsorption it is thus appropriate to analyze the resulting species and their location in relation to the support.

A common trait shared by the two simulations is that the hydroxyl group of the citrate remains intact for the whole sampling time, in line with its estimated ionization $\mathrm{p} K_{\mathrm{a}}$ (around 14.4) ${ }^{38}$ whereas the carboxyl protons are readily dissociable. In the first simulation, corresponding to a basic solution, the cit(-3) molecules, which are the only citrate species at the beginning of the simulation, are partially converted to other protonated species, namely $\operatorname{cit}(-3)(60 \%)$, Hcit $(-2)(30 \%)$, $\mathrm{H}_{2} \operatorname{cit}(-1)(10 \%)$. A similar behavior was previously suggested by a study of an aqueous solution of cysteine. ${ }^{39}$ There, the interactions at the water/air interface generated proton exchanges that lead to a local chemical composition quite different from the bulk. In the second simulation reported in the present study it is observed that starting from $100 \%$ of Hcit $(-2)$, which roughly represents an acidic solution, the equilibrium composition around the AuNP is predicted as cit(-3) (35\%), Hcit(-2) (40\%), $\mathrm{H}_{2} \operatorname{cit}(-1)(20 \%)$ and the appearance of completely protonated citric acid molecules (5\%) is also observed. This distribution of the species does not correspond to a definite $\mathrm{pH}$ suggesting that reference titration curves and $\mathrm{pH}$ cannot be used for these specific conditions, which are the results of local effects. Indeed, the analysis of the species indicates that speciation depends on the location of the molecules and is strongly influenced by their attachment to the AuNP. This is especially apparent in the case of acidic $\mathrm{pH}$. A similar behavior was observed previously for other systems, i.e. in the case of $\mathrm{TiO}_{2}$ nanoparticles ${ }^{\mathbf{4 0}}$ and for citrate adlayers on AuNPs through 

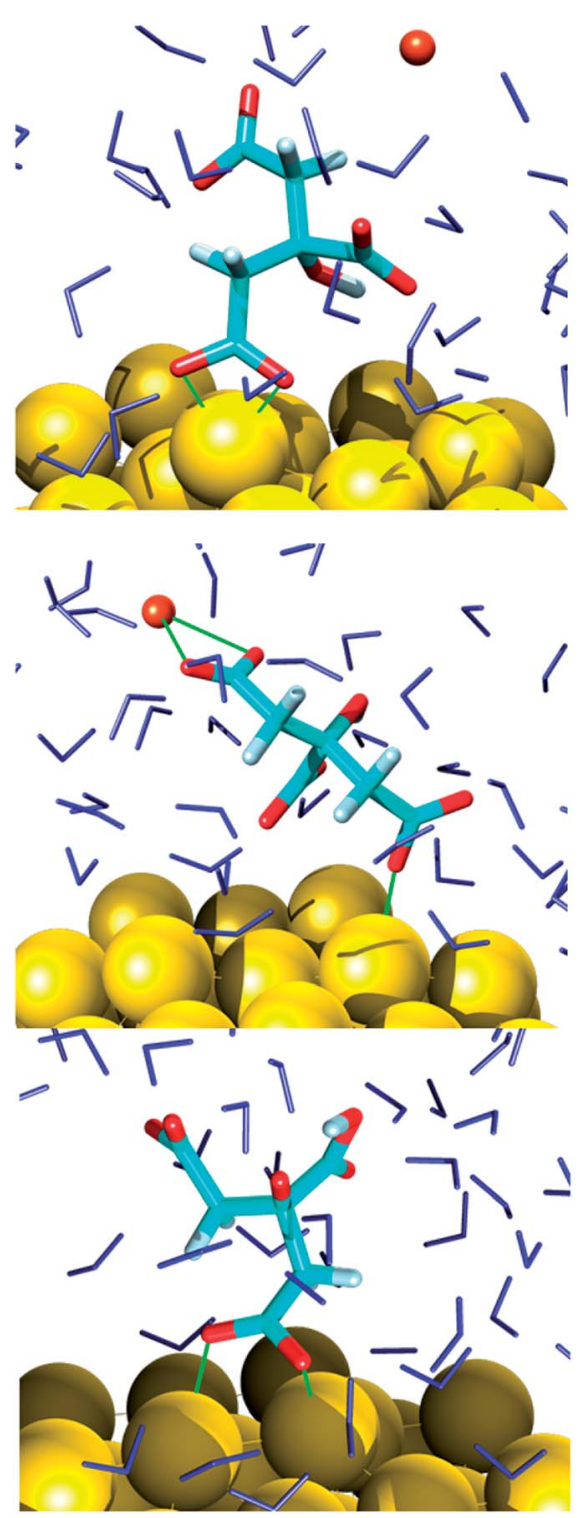

Fig. 1 Typical coordination modes of one carboxyl group to the gold interface. Configurations extracted from the final sampled geometry. Most of the surrounding molecules have been removed for clarity. $\mathrm{Na}^{+}$ ions are orange spheres and some of the water molecules surrounding the adsorbate are displayed as blue slim sticks. The green lines indicate distances lower then $2.6 \AA$. Au-O separations are around $2.3 \AA$.

FTIR and XPS analyses. ${ }^{41,42}$ To check that this behavior is due to the adsorption on the AuNP, we restricted the analysis of the molecular dynamics simulations to the citrate species farther from the nanoparticle, thus to an environment close to a pure solution. In this case the predicted ratios for citrate anions are in reasonable agreement with the experimental ones, specified by the titration curves, with a deviation of less than $10 \%$.

\section{Adsorption mode}

As far as the binding mode is concerned, $50 \%$ of the adsorbates were connected to the top gold layer through the coordination of just one of the terminus carboxyl group, whereas the other
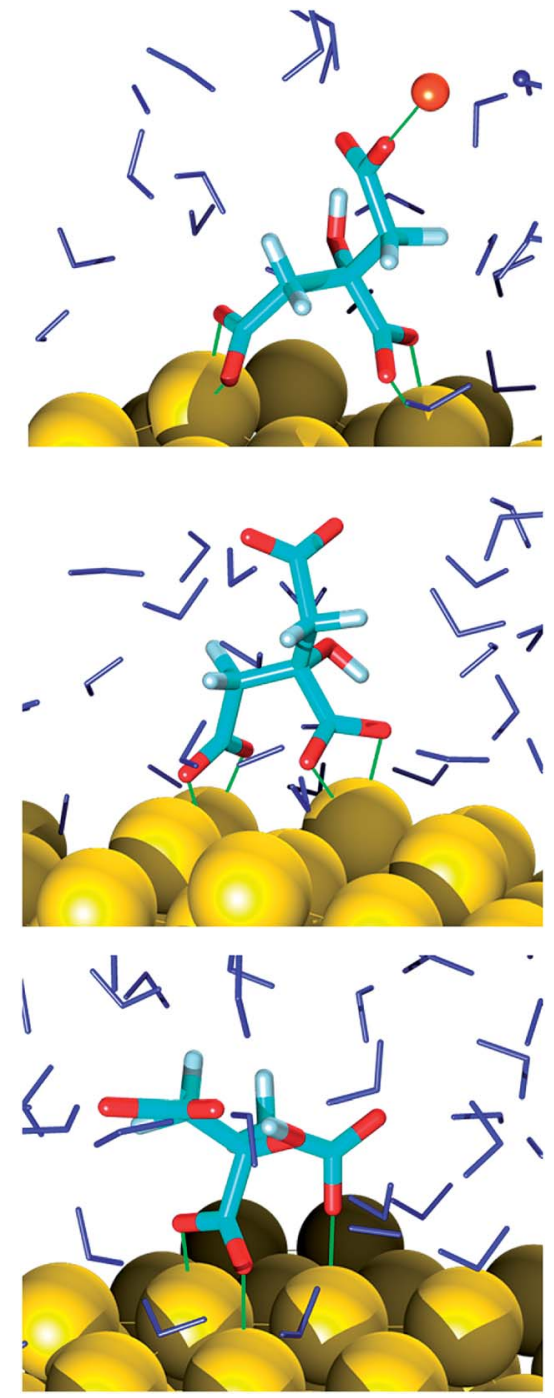

Fig. 2 Typical coordination modes of two carboxyl groups to the gold interface. Configurations extracted from the final sampled geometry. Most of the surrounding molecules have been removed for clarity. $\mathrm{Na}^{+}$ ions are orange spheres and some of the water molecules surrounding the adsorbate are displayed as blue slim sticks. The green lines indicate distances lower then $2.6 \AA$. Au-O separations are around $2.3 \AA$.

$50 \%$ were adsorbed through both the central and a terminus moieties, thus resulting in a stronger attachment to the metal support. Representative configurations of the adsorbed molecules are shown in Fig. 3-5. It was noticed that structures bound to the interface by means of all the three carboxyl units were rarely present and mainly consisted of chelate formations. A common arrangement is the one shown in the top right picture of Fig. 6. There, two carboxyl groups of the molecule (middle and terminus moieties) with the help of the hydroxyl oxygen entrap a gold adatom, while the other carboxyl terminus is coordinated through one of its oxygens to the surface. In the picture on the left hand side, instead, the enveloping mechanism is double but the connection of the citrate to the surface seems relatively weaker. Finally, the bottom picture of Fig. 6 shows the complex organization of the various adsorbates on the surface, the formed staple motifs (gold atoms captured by 


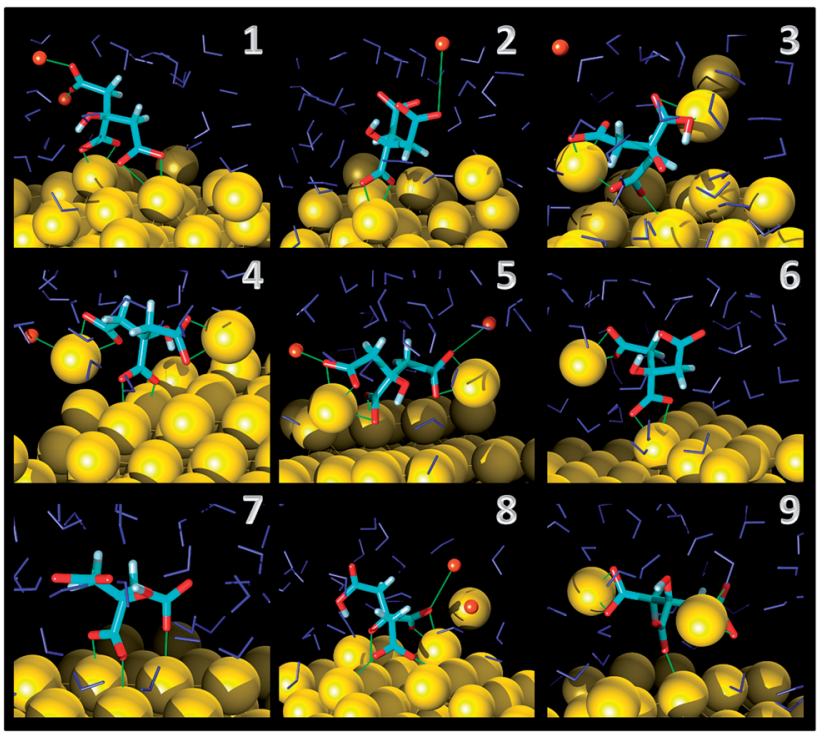

Fig. 3 Selected adsorbate configurations.

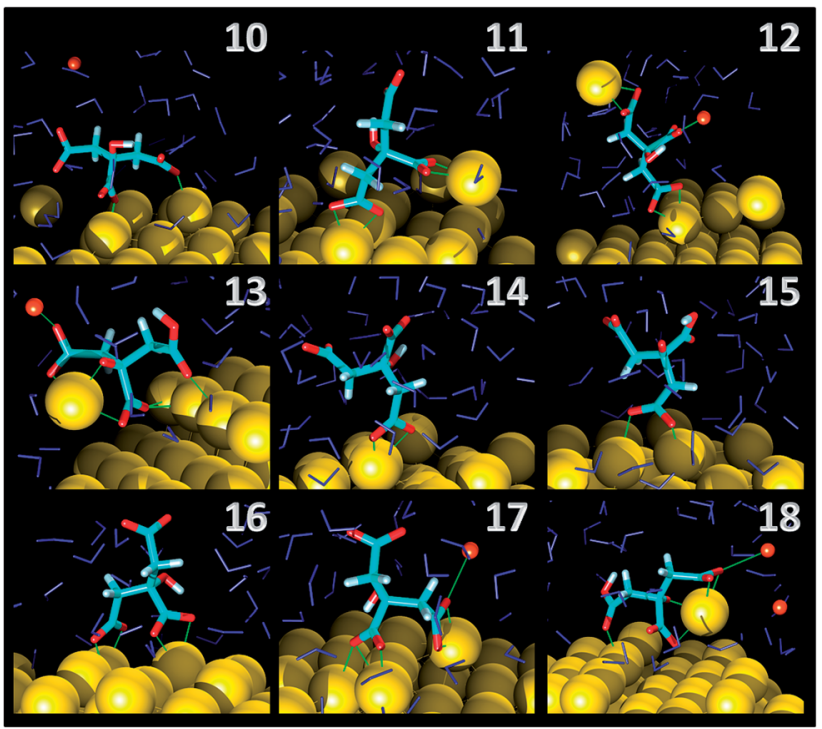

Fig. 4 Selected adsorbate configurations.

two different adsorbed molecules), interactions with ions, molecules in solution interacting with the deposited layer. This is just a small portion of a very complex scenario where many possible configurations are probable as demonstrated in several studies. ${ }^{41,43}$

Even though the dominant adsorption in both acidic and basic simulations was realized through one of the terminus carboxyl groups (with a probability of $85 \%$ and $75 \%$ for the acidic and basic cases, respectively), a slight reduction (to around $10 \%$ ) of these species and the simultaneous increase in the central group coordination (with a probability of $15 \%$ and $25 \%$ for the acidic and basic cases, respectively), was observed for acidic conditions. Most probably, the protonation of the terminus sites discouraged their binding to the surface but

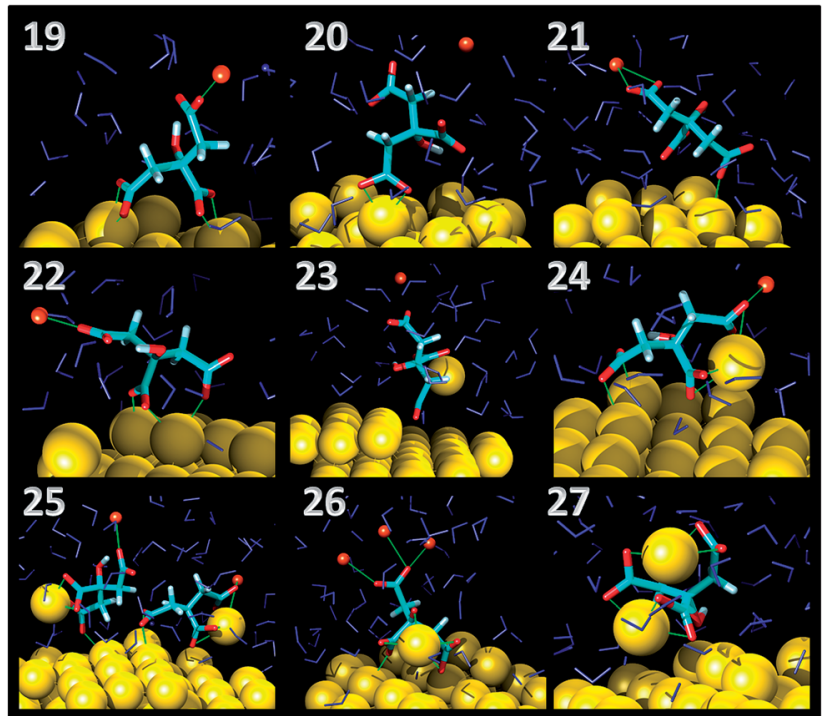

Fig. 5 Selected adsorbate configurations.

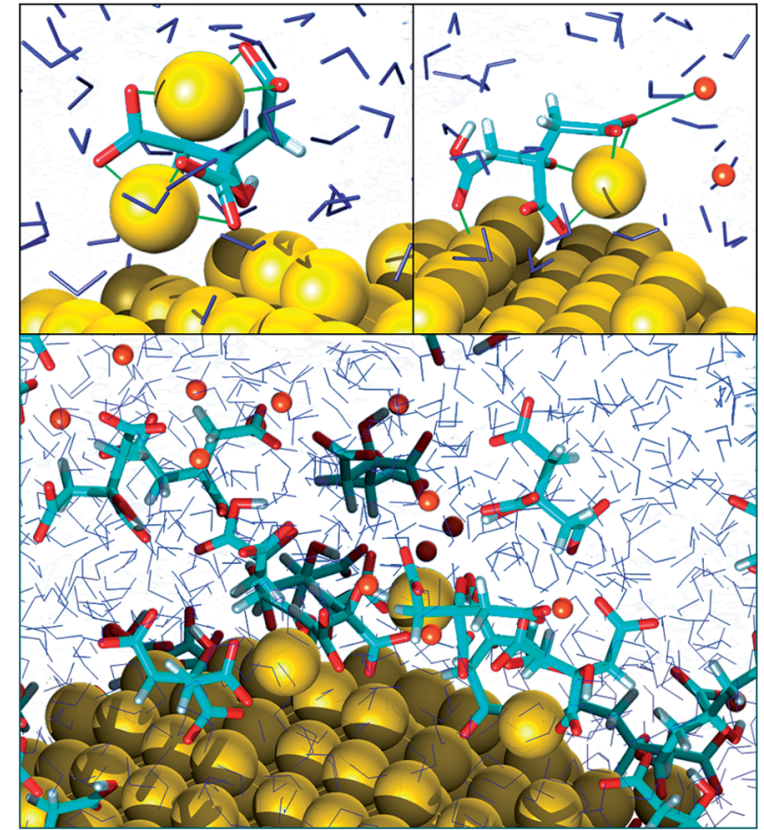

Fig. 6 Typical adsorption modes of chelating agents. Configurations extracted from the final sampled geometry. Most of the surrounding molecules have been removed for clarity. $\mathrm{Na}^{+}$ions are orange spheres and some of the water molecules surrounding the adsorbate are displayed as blue slim sticks. The green lines indicate distances lower then $2.6 \AA ̊$. Au-O separations are around $2.3 \AA$.

favored their extension towards the outer solution (solvent or citrate molecules far from the metal interface).

The complexity of the structural arrangement of the adsorbed citrate layers on AuNps was analyzed in various studies ${ }^{41,42}$ and it was concluded that many different schemes were possible simultaneously. It was proposed that the lowest $\mathrm{p} K_{\mathrm{a}}$ carboxyl group (middle moiety) plays a crucial role in the conformation of the adsorbed molecules and it was most 
preferentially bound to the surface by coordinating its carboxyl oxygens, while one of the other two terminus moieties could be adsorbed or not.

This is partially consistent with our results, which exclude the single binding option because statistically less significant. In fact, due to the conformational characteristics of this acid, i.e. the closeness of the terminus groups to the central moiety, there was a marked tendency to engage also one of the end groups in interactions with the surface. In the present simulations this cooperation (terminus + central) seemed more favorable, in agreement with other studies. ${ }^{\mathbf{4 4 - 4 6}}$ Furthermore, in line with the results of ref. 41 (see Fig. 6 and 3-5) part of the attached molecules extended the uncoordinated groups toward the neighboring species farther from the surface and formed interconnected networks. These new associations together with the captured adatom complexes determined an increase in the thickness of the coverage (which was around 7-8 $\AA$ ). The agreement with the data reported by J.-W. Park and J. S. Shumaker-Parry, ${ }^{\mathbf{4 1}}$ who estimated the thickness of the citrate layer on the basis of the interaction of the particles functionalized with alkanedithiol chains, is very good. They concluded that the minimum thickness could fall in a range around $6.5 \AA$ and that the effective depth was approximately 8-10 A.

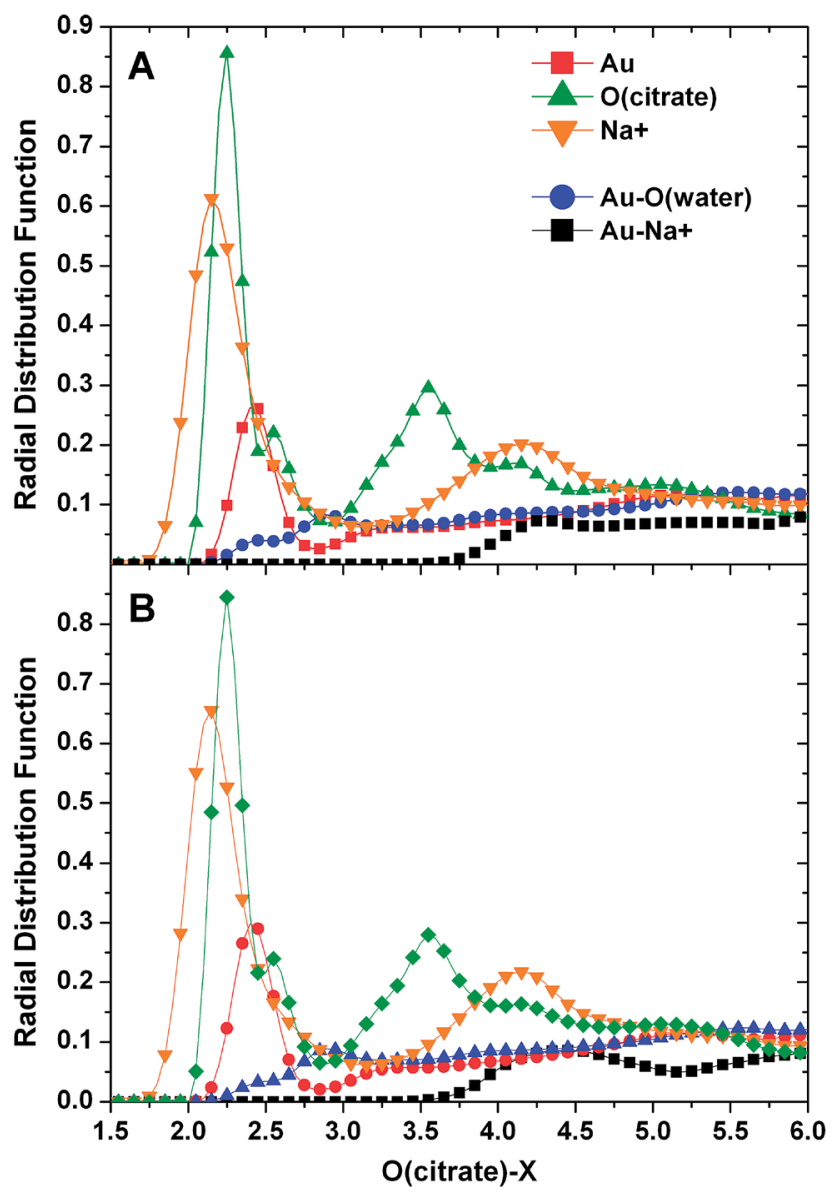

Fig. 7 Normalized atom-atom radial distribution functions of: citrate oxygens-citrate oxygens, citrate oxygens- $\mathrm{Au}$, citrate oxygens $-\mathrm{Na}^{+}$ $\mathrm{Au}-$ water oxygen, $\mathrm{Au}-\mathrm{Na}^{+}$. (A) Basic $\mathrm{pH}$ simulation. (B) Acidic $\mathrm{pH}$ simulation.
Examination of the atom-atom radial distribution functions of the citrate oxygens with all the other atoms of the system can be useful to compare the behavior of the molecule in the two different $\mathrm{pH}$ conditions, to check self-interactions and to determine the location of the adsorbate in relation to the support. The normalized RDF plots are shown in Fig. 7.

As it can be noticed, the trends obtained for the two simulations are strikingly similar suggesting that the observed population differences (discussed above) did not influence the binding behavior of the molecule. We could speculate that the final populations for the acidic conditions are influenced by the binding to the AuNP. The two $\mathrm{O}$ (citrate)-O(citrate) and $\mathrm{O}$ (citrate) $-\mathrm{Na}^{+}$sharp peaks centered at about $2.25 \AA$ confirmed self-interactions of the molecules and their coordination to sodium ions (evidenced also in most of the images depicting the adsorbates) independently of their location (i.e. in solution or on the AuNP surface). The lower O(citrate)-Au peak located at around $2.4 \AA$ indicates coordination to gold and its height suggests that only a portion of the molecules is stably adsorbed. Moreover, water oxygens and $\mathrm{Na}^{+}$ions remain farther from the
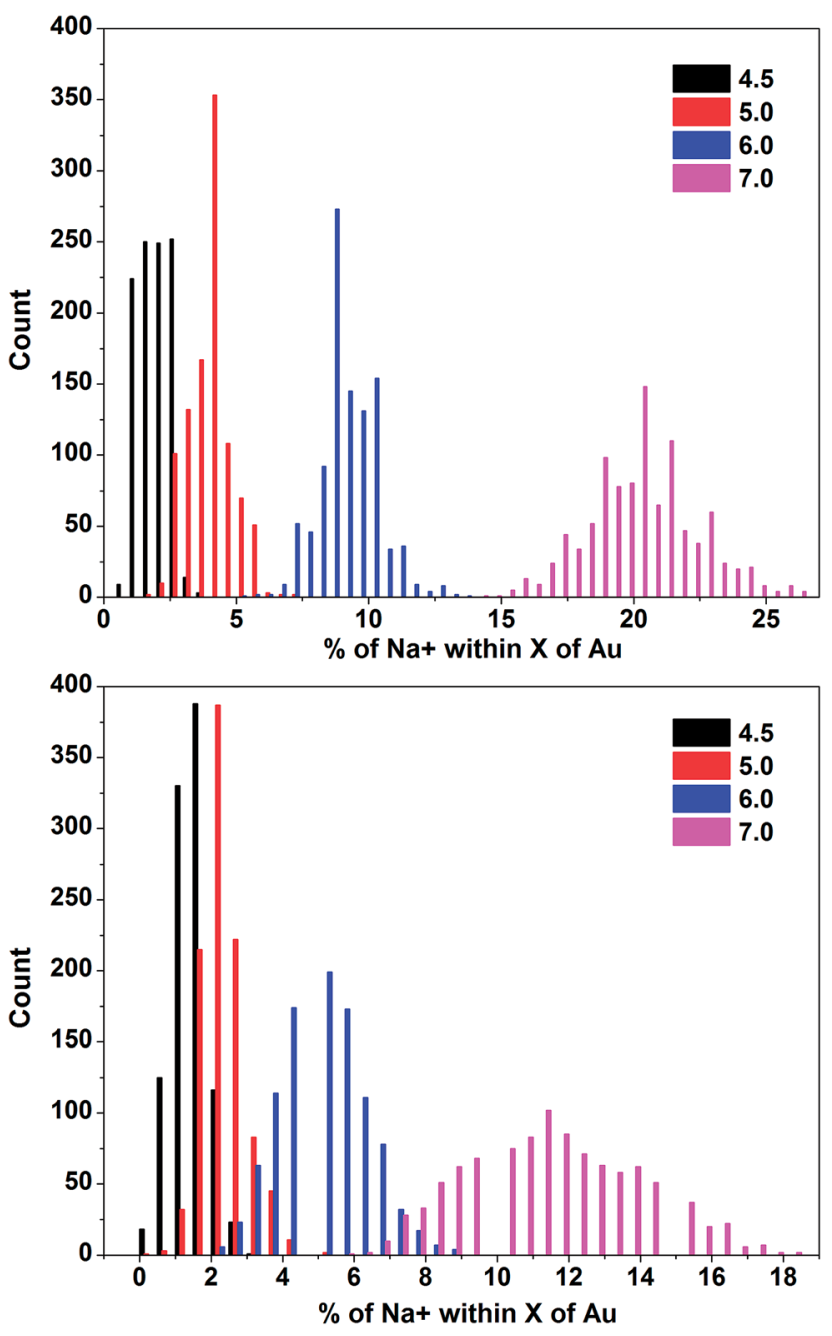

Fig. 8 Distributions of the percentage of $\mathrm{Na}^{+}$ions within 4.5, 5.0, 6.0 and $7.0 \AA$ of $\mathrm{Au}$. (Top) Basic pH simulation. (Bottom) Acidic pH simulation. 
surface in relation to the citrate oxygen (at 2.8 and $4.3 \AA$, respectively).

The strength of the adsorption on gold and the coordination of the carboxyl oxygens to $\mathrm{Na}^{+}$was estimated by inspecting the coordination numbers for $\mathrm{O}$ (citrate) $-\mathrm{Au}$ and $\mathrm{O}$ (citrate) $-\mathrm{Na}^{+}$ distances lower than $3 \AA$ and percentage of occupation of these states during the last $100 \mathrm{ps}$ of the production. In the case of gold, the result suggested that most probably (76\%) one or two $\mathrm{Au}$ atoms were found coordinated to the carboxyl oxygens in $80 \%$ of the configurations, whereas for $3 \mathrm{Au}$ atoms the probability decreased to $10 \%$ and the occupation of these states was also reduced (55\%).

These reactive simulations were capable of identifying a great variety of gold-citrate interactions ranging from weak to strong coupling, namely physisorption to chemisorption. However, it was not straightforward to discriminate between them just because many intermediate situations were present.
The results suggest that the strongest couplings were those where the Au-O distance was about 2.10-2.12 A, which could be identified with effective chemisorbed species. Then, slightly weaker adsorption were found for oxygen-Au distances in the range 2.23-2.30 $\AA$ (in Fig. $\mathrm{S} 3 \dagger$ is shown the distribution of $\mathrm{Au}-\mathrm{O}$ minimum distance).

As far as $\mathrm{Na}^{+}$is concerned, the probability of finding one or two ions within $3 \AA$ of the adsorbed citrate oxygen was $36 \%$ in the basic $\mathrm{pH}$ simulation but moderately lower (27\%) for the acid $\mathrm{pH}$, with a comparable occupation of $60 \%$.

In order to explore further the presence of the sodium ions in the first layer around the AuNP (between 4.5 and $7 \AA$ ) the distribution of the percentage of ions in different shells was calculated from the final 100 ps of the two simulations (Fig. 8). In line with the other data many more ions were found within $7 \AA$ of the AuNP in the basic simulation suggesting, as expected, that a more negatively charged environment is obtained in this case.
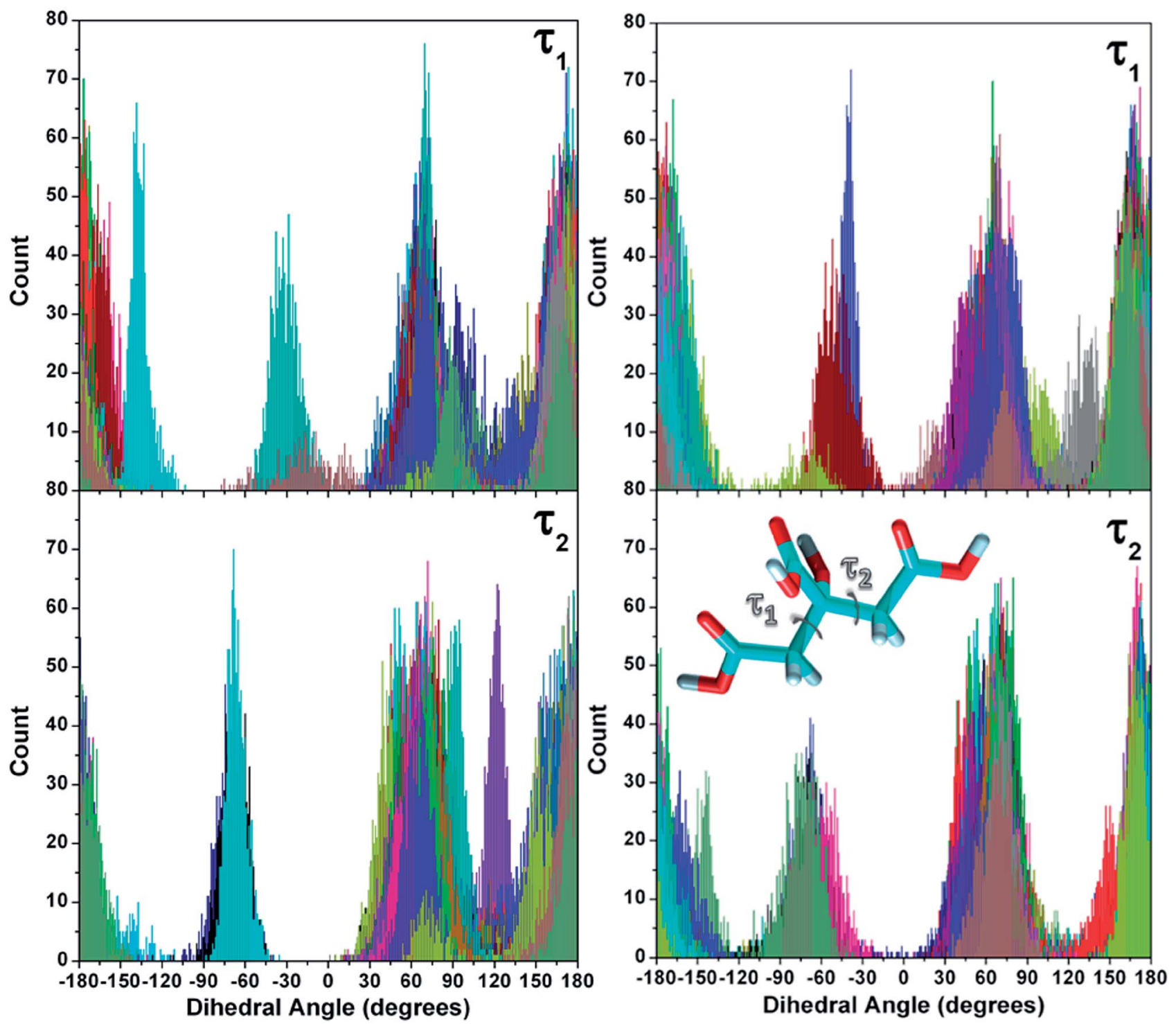

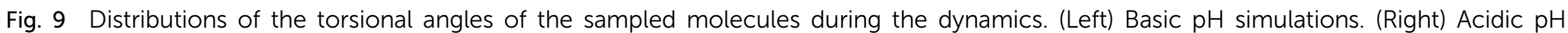
simulations. Different colors refer to different frames sampled from the last part of the production trajectory. 


\section{Molecular flexibility}

The conformational characteristics of the molecule were analyzed considering the two main torsional angles defining the position of the carboxyl groups (Fig. 9). These were investigated in another study ${ }^{47}$ to determine the conformers of citric acid in the gas phase and in solution and probe the effects due to different $\mathrm{pH}$ on its adsorption on $\mathrm{NaCl}$. The torsion distributions of the molecules sampled in this study are displayed in Fig. 9.

Comparison with the data of the minimum energy conformations identified in ref. 47 reveals a perfect agreement with the peak values of the distributions, suggesting that the adsorption was not influential in shaping the structures. As it can be noticed, all the angles are equally explored by the two simulations. This is also in agreement with the experimental NMR data reported in ref. 24 regarding the conformation of the carbon backbone. They confirm that citrate retains the structure and hydration arrangement even when bound to the AuNP surface and only the carboxylate groups interacting directly with gold are perturbed.

\section{Conclusion}

The results of this investigation regarding the adsorption of trisodium citrate on a gold nanoparticle at two different pHs, which were realized by a different concentration of the initial species present in the system, suggest that the whole stabilizing mechanism arises from a combination of different concerted events. First, the direct adsorption of the molecule on the surface of the AuNP in a preferential fully deprotonated form confers stability to the metal support by altering its surface charge. The adsorption induces a modulation of the local conditions, which do not correspond any longer to the tabulated values for a pure solution. The adsorption of the citrate consists of a strong coordination of the carboxyl oxygens to the surface that can involve one or more groups. Second, the chelating ability of the partially adsorbed molecules prevents the migration of the gold atoms emerged from the surface. These adatoms are entrapped in the covering layer and remain close to the nanoparticle interface. Consequently, citrate limits the extension of the surface reconstruction. Third, several citrate molecules on the interface are found interconnected through metal adatoms. These are characteristic substructures, named staple motifs, were already observed in the case of cysteine and cystine adsorption. ${ }^{32,33}$ Fourth, $\mathrm{Na}^{+}$ions are found within the adsorbed monolayer in a region between 4-7 $\AA$ from the gold substrate in line with experiment.

\section{Conflicts of interest}

There are no conflicts to declare.

\section{Acknowledgements}

S. M. is grateful to Adri C. T. van Duin for the stand-alone version of ReaxFF and his support.

\section{References}

1 X. Yang, M. Yang, B. Pang, M. Vara and Y. Xia, Gold Nanomaterials at Work in Biomedicine, Chem. Rev., 2015, 115, 10410-10488.

2 N. S. Abadeer and C. J. Murphy, Recent Progress in Cancer Thermal Therapy Using Gold Nanoparticles, J. Phys. Chem. C, 2016, 120, 4691-4716.

3 C. R. Maldonado, L. Salassa, N. Gomez-Blanco and J. C. Mareque-Rivas, Nanofunctionalization of Metal Complexes for Molecular Imaging and Anticancer Therapy, Coord. Chem. Rev., 2013, 257, 2668-2688.

4 S. Hwang, J. Nam, S. Jung, J. Song, H. Doh and S. Kim, Gold nanoparticle-mediated photothermal therapy: current status and future perspective, Nanomedicine, 2014, 9, 2003-2022.

5 N. Panwar, C. Yang, F. Yin, H. S. Yoon, T. S. Chuan and K.-T. Yong, Rnai-Based Therapeutic Nanostrategy: il-8 Gene Silencing in Pancreatic Cancer Cells using Gold Nanorods Delivery Vehicles, Nanotechnology, 2015, 26, 365101-365115.

6 K. C. Black, N. D. Kirkpatrick, T. S. Troutman, L. Xu, J. Vagner, R. J. Gillies, J. K. Barton, U. Utzinger and M. Romanowski, Gold Nanorods Targeted to Delta Opioid Receptor: Plasmon-Resonant Contrast and Photothermal Agents, Mol. Imaging, 2008, 7, 50-57.

7 E. E. Bedford, S. Boujday, C. M. Pradierab and F. X. Gu, Nanostructured and Spiky Gold in Biomolecule Detection: Improving Binding Efficiencies and Enhancing Optical Signals, RSC Adv., 2015, 5, 16461-16475.

$8 \mathrm{~A}$. Li and S. Li, Large-Volume Hot Spots in Gold Spiky Nanoparticle Dimers for High-Performance SurfaceEnhanced Spectroscopy, Nanoscale, 2014, 6, 12921-12928.

9 A. Foubert, N. V. Beloglazova and S. D. Saeger, Comparative Study of Colloidal Gold and Quantum Dots as Labels for Multiplex Screening Tests for Multi-Mycotoxin Detection, Anal. Chim. Acta, 2017, 955, 48-57.

10 J.-Y. Yoon, G.-S. Choi, I.-S. Cho and S.-K. Choi, Development of Rapid Immune-gold Strip Kit for On-Site Diagnosis of Tomato Spotted Wilt Virus, Res. Plant Dis., 2014, 20, 15-20.

11 B. Ahmad, N. Hafeez, S. Bashir, A. Rauf and A. Mujeeb-UrRehman, Phytofabricated Gold Nanoparticles and their Biomedical Applications, Biomed. Pharmacother., 2017, 89, 414-425.

12 S. Her, D. A. Jaffray and C. Allen, Gold nanoparticles for applications in cancer radiotherapy: Mechanisms and recent advancements, Adv. Drug Delivery Rev., 2017, 109, 84-101.

13 M. Patil and G.-D. Kim, Eco-friendly Approach for Nanoparticles Synthesis and Mechanism behind Antibacterial Activity of Silver and Anticancer Activity of Gold Nanoparticles, Appl. Microbiol. Biotechnol., 2017, 101, 79-92.

14 S. Rosa, C. Connolly, G. Schettino, K. Butterworth and K. Prise, Biological Mechanisms of Gold Nanoparticle Radiosensitization, Cancer Nanotechnol., 2017, 8, 1-25.

15 N. Goswami, Q. Y. T. Chen and J. Xie, Mechanistic Exploration and Controlled Synthesis of Precise ThiolateGold Nanoclusters, Coord. Chem. Rev., 2016, 329, 1-15. 
16 E. Carbó-Argibay and B. Rodríguez-González, Controlled Growth of Colloidal Gold Nanoparticles: Single-Crystalline versus Multiply-Twinned Particles, Isr. J. Chem., 2016, 56, 214-226.

17 A. Marsico, S. G. Elci, D. F. Moyano, G. Y. Tonga, B. Duncan, R. F. Landis, V. M. Rotello and R. W. Vachet, Enhanced Laser Desorption/Ionization Mass Spectrometric Detection of Gold Nanoparticles in Biological Samples Using the Synergy Between Added Matrix and the Gold Core, Anal. Chem., 2015, 87, 12145-12150.

18 M. R. Esfahani, V. L. Pallema, H. A. Stretz and M. J. M. Wells, Extinction, Emission, and Scattering Spectroscopy of 5$50 \mathrm{~nm}$ Citrate-Coated Gold Nanoparticles: An Argument for Curvature Effects on Aggregation, Spectrochim. Acta, Part A, 2017, 175, 100-109.

19 Y.-C. Yeh, B. Creran and V. M. Rotello, Gold Nanoparticles: Preparation, Properties, and Applications in Bionanotechnology, Nanoscale, 2012, 4, 1871-1880.

20 S. H. Brewer, W. R. Glomm, M. C. Johnson, M. K. Knag and S. Franzen, Probing BSA Binding to Citrate-Coated Gold Nanoparticles and Surfaces, Langmuir, 2005, 21, 9303-9307.

21 M. Stobiecka, M. Hepel and K. Coopersmith, Resonance Elastic Light Scattering (RELS) Spectroscopy of Fast NonLangmuirian Ligand-Exchange in Glutathione-Induced Gold Nanoparticle Assembly, J. Colloid Interface Sci., 2010, 350, 168-177.

22 M.-C. Daniel and D. Astruc, Gold Nanoparticles: Assembly, Supramolecular Chemistry, Quantum-Size-Related Properties, and Applications toward Biology, Catalysis, and Nanotechnology, Chem. Rev., 2004, 104, 293-346.

23 T. C. Preston, M. Nuruzzaman, N. D. Jones and S. Mittler, Role of Hydrogen Bonding in the pH-Dependent Aggregation of Colloidal Gold Particles Bearing SolutionFacing Carboxylic Acid Groups, J. Phys. Chem. C, 2009, 113, 14236-14244.

24 H. Al-Johani, E. Abou-Hamad, A. Jedidi, C. M. Widdifield, J. Viger-Gravel, S. S. Sangaru, D. Gajan, D. H. Anjum, S. Ould-Chikh, M. N. Hedhili, et al., The Structure and Binding Mode of Citrate in the Stabilization of Gold Nanoparticles, Nat. Chem., 2017, 9, 890-895.

25 A. C. T. van Duin, J. M. A. Baas and B. van de Graaf, Delft Molecular Mechanics: A New Approach to Hydrocarbon Force Fields. Inclusion of a Geometry-Dependent Charge Calculation, J. Chem. Soc., Faraday Trans., 1994, 90, 28812895.

26 K. Joshi, A. C. T. van Duin and T. Jacob, Development of a ReaxFF Description of Gold Oxides and Initial Application to Cold Welding of Partially Oxidized Gold Surfaces, J. Mater. Chem., 2010, 20, 10431-10437.

27 J. A. Keith, D. Fantauzzi, T. Jacob and A. C. T. van Duin, Reactive Forcefield for Simulating Gold Surfaces and Nanoparticles, Phys. Rev. B: Condens. Matter Mater. Phys., 2010, 81, 235404.

28 T. T. Järvi, A. C. T. van Duin, K. Nordlund and W. A. Goddard III, Development of Interatomic ReaxFF Potentials for Au-S-C-H Systems, J. Phys. Chem. C, 2011, 115, 10315-10322.
29 S. Monti, A. Corozzi, P. Fristrup, K. L. Joshi, Y. K. Shin, P. Oelschlaeger, A. C. T. van Duin and V. Barone, Exploring the Conformational and Reactive Dynamics of Biomolecules in Solution using an Extended Version of the Glycine Reactive Force Field, Phys. Chem. Chem. Phys., 2013, 15, 15062-15077.

30 X. Li, V. Carravetta, C. Li, S. Monti, Z. Rinkevicius and H. Ågren, Optical Properties of Gold Nanoclusters Functionalized with a Small Organic Compound: Modeling by an Integrated Quantum-Classical Approach, J. Chem. Theory Comput., 2016, 12, 3325-3339.

31 S. Monti, V. Carravetta and H. Ågren, Simulation of Gold Functionalization with Cysteine by Reactive Molecular Dynamics, J. Phys. Chem. Lett., 2016, 7, 272-276.

32 S. Monti, V. Carravetta and H. Ågren, Decoration of Gold Nanoparticles with Cysteine in Solution: Reactive Molecular Dynamics Simulations, Nanoscale, 2016, 8, 12929-12938.

33 S. Monti, V. Carravetta and H. Ågren, Theoretical Study of the Adsorption Mechanism of Cystine on $\mathrm{Au}(110)$ in Aqueous Solution, Small, 2016, 12, 6134-6143.

34 J. Schubert, in Ion Exchange, ed. F. C. Nachod, Academic Press, 1949, pp. 167-222.

35 H. J. C. Berendsen, J. P. M. Postma, W. F. V. Gunsteren, A. DiNola and J. R. Haak, Molecular Dynamics with Coupling to an External Bath, J. Chem. Phys., 1984, 81, 3684-3690.

36 E. J. Baerends, et al., ADF, adf2016.106. SCM, Theoretical Chemistry, Vrije Universiteit, Amsterdam, The Netherlands, http://www.scm.com.

37 S. Plimpton, Fast Parallel Algorithms for Short-Range Molecular-Dynamics, J. Comput. Phys., 1995, 117, 1-19.

38 A. M. N. Silva, X. Kong and R. C. Hider, Determination of the pKa value of the hydroxyl group in the $\alpha$-hydroxycarboxylates citrate, malate and lactate by 13C NMR: implications for metal coordination in biological systems Molecular Dynamics with Coupling to an External Bath, BioMetals, 2009, 22, 771-778.

39 Á. M. da Silva, A. Mocellin, S. Monti, C. Li, R. R. T. Marinho, A. Medina, H. Agren, V. Carravetta and A. N. de Brito, Surface-Altered Protonation Studied by Photoelectron Spectroscopy and Reactive Dynamics Simulations, J. Phys. Chem. Lett., 2015, 6, 807-811.

40 I. A. Mudunkotuwa and V. H. Grassian, Citric Acid Adsorption on TiO2 Nanoparticles in Aqueous Suspensions at Acidic and Circumneutral pH: Surface Coverage, Surface Speciation, and Its Impact on Nanoparticle-Nanoparticle Interactions, J. Am. Chem. Soc., 2010, 132, 14986-14994.

41 J. W. Park and J. S. Shumaker-Parry, Structural Study of Citrate Layers on Gold Nanoparticles: Role of Intermolecular Interactions in Stabilizing Nanoparticles, $J$. Am. Chem. Soc., 2014, 136, 1907-1921.

42 M. R. Esfahani, V. L. Pallem, H. A. Stretz and M. J. Wells, Extinction, Emission, and Scattering Spectroscopy of 5$50 \mathrm{~nm}$ Citrate-Coated Gold Nanoparticles: An Argument for Curvature Effects on Aggregation, Spectrochim. Acta, Part A, 2017, 175, 100-109. 
43 J. Piella, N. G. Bastús and V. Puntes, Size-controlled synthesis of sub-10-nanometer citrate-stabilized gold nanoparticles and related optical properties, Chem. Mater., 2016, 28, 1066-1075.

44 G. Mpourmpakis and D. G. Vlachos, Growth Mechanisms of Metal Nanoparticles via First Principles, Phys. Rev. Lett., 2009, 102, 155505.

45 M. A. Uppal, A. Kafizas, M. B. Ewinga and I. P. Parkin, The Effect of Initiation Method on the Size, Monodispersity and Shape of Gold Nanoparticles formed by the Turkevich Method, New J. Chem., 2010, 34, 2906-2914.

46 Q. Zhang, N. Li, J. Goebl, Z. Lu and Y. Yin, A systematic Study of the Synthesis of Silver Nanoplates: is Citrate a magic reagent?, J. Am. Chem. Soc., 2011, 133, 18931-18939.

47 M. A. S. Khan and S. A. G. Bishwajit, Probing the Influence of pH Dependent Citric Acid towards the Morphology of Rock Salt: a Computational Study, CrystEngComm, 2009, 11, 2660-2667. 